\title{
Atypical Presentation of Neuroleptic Malignant Syndrome in a Pediatric Patient
}

\section{Abstract}

This is a case of a 16-year-old male with a history of attention deficit hyperactivity disorder (ADHD), oppositional defiant disorder (ODD), obsessive compulsive disorder (OCD), and bipolar disorder (BD) who presented to the emergency department with hypertension, tachycardia, rigidity, and altered mental status 2 months after the initiation of olanzapine. Symptoms were most consistent with Neuroleptic Malignant Syndrome (NMS). Neuroleptic Malignant Syndrome (NMS) a potentially fatal neurologic emergency generally precipitated by the use of neuroleptic agents. The clinical syndrome is characterized by mental status changes, fever, dysautonomia, and rigidity. While most cases of NMS are precipitated by "typical" neuroleptic agents such as haloperidol and fluphenazine other agents such as lower potency or "atypical" antipsychotic drugs have been implicated in the development of NMS as well. To our knowledge, this is the first reported pediatric case of NMS attributed by atypical or second-generation antipsychotics. Additionally, our patient did not exhibit the classic tetrad of clinical symptoms on presentation.

Key Words: neuroleptic malignant syndrome, atypical antipsychotics, second generation antipsychotics, olanzapine

Received: April 13, 2017; Accepted: April 25, 2017; Published: April 30, 2017

\section{Introduction}

Neuroleptic Malignant Syndrome (NMS) is an uncommon but potentially fatal reaction that is known to occur with typical or first-generation antipsychotics [1]. The association of NMS with atypical or second-generation antipsychotics is less commonly documented. Moreover, atypical presentations of NMS, such as the absence of fever or rigidity and a slowly evolving clinical course over a period of months, can further confound its ability to be recognized and can lead to a significant delay in appropriate treatment [2].

\section{Case Presentation}

A 16-year-old teenager with a history of attention deficit hyperactivity disorder (ADHD), oppositional defiant disorder (ODD), obsessive compulsive disorder (OCD), and bipolar disorder (BD) presented to the emergency department with symptoms of altered mental status and diaphoresis immediately after discharge from an inpatient behavioral rehabilitation center where a slow, up-titration of olanzapine was started 2 months prior. Additionally, in the week prior to presentation there had been a reported, more rapid increase in the nightly dose of
Yaphockun $\mathrm{K}^{1}$, Corbett $\mathrm{B}^{2}$ and Conrad $\mathrm{H}^{3}$

1 Department of Emergency Medicine, Rady Children's Hospital, University of California, San Diego, California, USA

2 Department of Toxicology, Rady Children's Hospital, University of California, San Diego, California, USA

3 Department of Emergency Medicine, Rady Children's Hospital, University of California, San Diego, California, USA

Corresponding author: Karen Yaphockun

” kyaphockun@rchsd.org

Department of Emergency Medicine, Rady Children's Hospital, University of California, 3020 Children's Way MC 5075, San Diego, CA 92123, California, USA.

Tel: $858-966-8036$

Fax: 858-966-7433

Citation: Yaphockun K, Corbett B, Conrad H. Atypical Presentation of Neuroleptic Malignant Syndrome in a Pediatric Patient. J Pediatr Care. 2017, 3:2.

olanzapine, although the exact dose change and time interval could not be recalled. On presentation, his medications included olanzapine (15 mg in the morning and $5 \mathrm{mg}$ at bedtime) and atomoxetine (60 $\mathrm{mg}$ once daily in the morning). Discontinued medications within the past 2 months included guanfacine, depakote, and aripiprazole.

On physical exam, the patient had a heart rate of 116 beats per minute, blood pressure of $154 / 87 \mathrm{mmHg}$, respiratory rate of 22 breaths per minute, and an oral temperature of $37.1^{\circ} \mathrm{C}$. He was diaphoretic, tremulous, and had dry oral mucosa. He answered questions appropriately but with delayed responses and intermittent stammering speech. The patient's pupils were symmetrically dilated but reactive. His neurologic exam revealed bradykinesia, cogwheel rigidity on range of motion testing of his shoulder and hips, and a shuffling gait. His rigidity was more notable in his lower extremities than his upper extremities. The remainder of his physical exam was unremarkable. 
Initial laboratory evaluation included a complete blood count, comprehensive metabolic panel, creatinine kinase, and coagulation panel, all of which were normal. Urine drug immunoassay testing for amphetamine/methamphetamine, barbiturates, benzodiazepines, cocaine metabolite, methadone, opiates, PCP, and cannabinoids was negative. Blood ethanol, acetaminophen and salicylate levels were also undetectable.

Based on his history of recent antipsychotic administration and up-titration of dose, clinical findings of autonomic instability, altered mental status, and rigidity, he was diagnosed with atypical neuroleptic malignant syndrome. In the emergency department, he was given a dose of lorazepam $2 \mathrm{mg}$ IV with subsequent improvement of agitation, tachycardia, and hypertension. Toxicology was consulted and given near complete resolution of symptoms with lorazepam, additional treatment was not initiated from the emergency department. The patient was admitted to the inpatient team who discontinued olanzapine and atomoxetine. He received a second dose of lorazepam $2 \mathrm{mg}$ IV for agitation on hospital day (HD) 2. By HD3, he was symptomfree and back to neurological baseline with complete resolution of tachycardia, hypertension, and rigidity. Although the initiation of bromocriptine was discussed, it was not incorporated in his treatment course, given the mild nature of his case. He remained afebrile throughout his inpatient course and was then discharged home.

\section{Discussion}

NMS is a potentially life-threatening emergency which is associated with exposure to a dopamine antagonist or withdrawal from a dopamine agonist; it is classically composed of a tetrad of symptoms including mental status changes, muscular rigidity, hyperthermia, and sympathetic nervous system lability in the absence of another cause [3]. The risks of morbidity and mortality from NMS are substantial and are secondary to systemic complications from dysautonomia. Although treatment for NMS is primarily supportive, early identification and prompt intervention is nonetheless lifesaving. Despite the need for early recognition, there is no diagnostic testing that exists for NMS. Historically, a spectrum-based concept has been considered for identification of NMS [2]. Demonstration of atypical presentations with a slow progression of symptoms or the absence of the key clinical signs of hyperthermia or muscular rigidity has also been published. Whether these cases are truly an atypical presentation or an early prodrome of NMS is unclear. Only recently has there been a diagnostic criteria developed by an expert consensus and even this has not yet been clinically validated. The newly proposed consensus neuroleptic malignant syndrome diagnostic criteria take into account common clinical signs and laboratory testing results performed in the evaluation of NMS (Table 1). Unique from previously synthesized criteria sets, in the consensus diagnostic criteria, the score given to a particular criterion reflects its relative importance. For example, when NMS is suspected, elicited history of the exposure to a dopamine antagonist or removal of a dopamine agonist receives a score of 20 while a negative laboratory work-up for other causes of signs and symptomatology receives a score of 7 . The total score is of 100 . As of yet, there is no cut-off point established for the diagnosis of NMS and as previously mentioned, this still requires validation [3].

This patient's diagnosis is complicated by the fact that he was on an atypical antipsychotic, olanzapine. While the exact cause of NMS is not known, the dominant theory is that central dopamine receptor blockade causes disruption in the central nervous system. Because of the strong dopamine-blocking ability of first generation antipsychotics, NMS has been recognized as a complication of this drug class. This association between typical antipsychotics and NMS has been described since 1960 with haloperidol [4]. With the decreased dopamine-blocking potential of second-generation antipsychotics, it was suspected that these agents were less likely to cause NMS. Previous case reports, however, have shown that clozapine, risperidone, olanzapine, and

Table 1 Neuroleptic malignant syndrome diagnostic criteria: Expert panel consensus.

\begin{tabular}{|c|c|}
\hline Diagnostic Criterion & Priority Score \\
\hline Exposure to dopamine antagonist, or dopamine agonist withdrawal within past 72 hours & 20 \\
\hline Hyperthermia $\left(>100.4^{\circ} \mathrm{F}\right.$ or $>38.0^{\circ} \mathrm{C}$ on at last 2 occasions, measured orally) & 18 \\
\hline Rigidity & 17 \\
\hline Mental status alteration (reduced or fluctuating level of consciousness) & 13 \\
\hline Creatine kinase elevation (at least 4 times the upper limit of normal) & 10 \\
\hline \multicolumn{2}{|l|}{ Sympathetic nervous system lability, defined as at last 2 of the following: } \\
\hline \multicolumn{2}{|l|}{ Blood pressure elevation (systolic or diastolic $\geq 25 \%$ above baseline) } \\
\hline \multicolumn{2}{|l|}{ Blood pressure fluctuation ( $\geq 20 \mathrm{mmHg}$ diastolic change or $\geq 25 \mathrm{mmHg}$ systolic } \\
\hline \multicolumn{2}{|l|}{ change within 24 hours) } \\
\hline \multirow{2}{*}{\multicolumn{2}{|c|}{$\begin{array}{l}\text { Diaphoresis } \\
\text { Urinary incontinence }\end{array}$}} \\
\hline & \\
\hline $\begin{array}{c}\text { Hyper metabolism, defined as a heart-rate increase ( } \geq 25 \% \text { above baseline) AND respiratory-rate increase ( } \geq 50 \% \\
\text { above baseline) }\end{array}$ & 5 \\
\hline Negative work-up for infectious, toxic, metabolic, or neurologic causes & 7 \\
\hline Total & 100 \\
\hline
\end{tabular}


quetiapine all have been implicated in NMS. In our case however not only was the patient taking atypical antipsychotics, but he also did not exhibit all clinical signs of NMS as he lacked elevated temperature. Other agents to consider as causes for NMS include antiemetic agents such as prochlorperazine and chlorpromazine. These antiemetics fall under the family of phenothiazines, which were among the first of the typical generation antipsychotics and, as we have previously mentioned, have dopamine-blocking effects. Another causative factor for the precipitation of NMS includes abrupt discontinuation of dopamine agonists. Also, it is important to consider that our patient was on olanzapine which is an atypical antipsychotic but was in the midst of titrating up his dose. It has been described that a recent dose increase or initiation of antipsychotics can precipitate NMS. Other suggested risk factors which precipitate NMS include higher doses of neuroleptics, greater changes in dose increments over a short period of time, parenteral administration of such medications, and simultaneous use of two neuroleptics [5].

Management of NMS first and foremost involves the removal of the causative factor. Thus, if a patient develops NMS as a result of initiation of a dopamine antagonist it must be discontinued. Additionally, if NMS is precipitated by discontinuation of a dopamine agonist this medication must be re-initiated. The next steps of treatment can include supportive management and, if needed, electroconvulsive therapy (ECT). Supportive treatment includes mechanisms of cooling for hyperthermia, volume repletion for insensible losses to maintain adequate renal perfusion and prevent renal failure from rhabdomyolysis, ventilatory support for those who develop respiratory failure, and pressor or antiarrhythmic support for those who develop cardiogenic complications. Pharmacologic treatment includes dantrolene to function as a muscle relaxant and dopamine agonists such as bromocriptine to re-establish dopaminergic tone. The role of benzodiazepines in NMS is primarily supportive in the management of NMS. Benzodiazapines decrease agitation and help to temper the sympathetic output that causes symptoms like hypertension and tachycardia. ECT is reserved as a last line treatment in patients who do not respond to pharmacologic therapy, exhibit pervasive catatonia, or have an underlying psychiatric diagnosis [5].

Morbidity from NMS is primarily due to renal failure from rhabdomyolysis. Thromboembolism secondary to immobilization, hypovolemia, and rigidity is also seen. Respiratory failure may also be seen and is due to chest wall rigidity. Cardiac complications come from dysautonomia. If the patient survives these lifethreatening complications, movement disorders like catatonia and parkinsonian symptoms may continue to persist for months after the acute symptoms resolve [5].

\section{Conclusion}

Critical points discussed in this article include clinical variability in the presentation of NMS and the absence of a validated gold standard in diagnosis. As exemplified by our patient, it is important to keep a high index of suspicion for NMS in patients who are on atypical antipsychotic medications especially if up titration of dosages is in process, as immediate intervention prevents significant morbidity and mortality. Although management generally consists of discontinuation of the inciting agent and symptomatic treatment, severe cases have led to the use of ECT. Our patient is an example of an atypical presentation, but a high clinical index of suspicion of an atypical antipsychotic causing such symptoms led to early recognition and intervention, all of which led to an improved clinical outcome.

\section{Disclaimer}

In compliance with the request of the Privacy Board of our institution, the designated sex in this case report has been arbitrarily assigned. 


\section{References}

1 Levenson JL (1985) Neuroleptic malignant syndrome. Am J Psychiatry 142: 1137-1145.

2 Picard LS, Lindsay S, Strawn JR, Kaneria RM, Patel NC, et al. (2008) Atypical neuroleptic malignant syndrome: Diagnostic controversies and considerations. Pharmacotherapy 2008: 530-535.

3 Gurrera RJ, Caroff SN, Cohen A (2010) An international consensus study of neuroleptic malignant syndrome diagnostic criteria using the delphi method. J Clin Psychiatry 72: 1222-1228.

4 Reeves RR, Torres RA, Liberto V, Hart RH (2002) Atypical neuroleptic malignant syndrome associated with olanzapine. Pharmacotherapy 22: 641-644.

5 Bhanushali MJ, Tuite PJ (2004) The evaluation and management of patients with neuroleptic malignant syndrome. Neurol Clin 22: 389-411. 